\title{
$\bullet \cdot$ \\ IJCRR \\ Section: Healthcare \\ Characterisation of Mutations Associated with Rifampicin Resistance in Patients Attending a Tertiary Care Centre, Kerala
}

ISI Impact Factor (2019-20): 1.628

IC Value (2019): 90.81 SJIF (2020) $=7.893$

(c) (i) (8)

Copyright@IJCRR

\section{Sreeja Nair ${ }^{1}$, Seema Oommen², Vidya Pai $^{3}$}

'Assistant Professor, Department of Microbiology, Pushpagiri Institute of Medical Sciences and Research Centre, Kerala, India; ${ }^{2}$ Specialist Microbiologist, Lifecare Hospital, and Molecular Biologist, Burjeel Medical City, Abu Dhabi, United Arab Emirates; ${ }^{P}$ rofessor and Head, Department of Microbiology, Yenepoya Medical College, Mangalore, India.

\section{ABSTRACT}

Introduction: Resistance to rifampicin is considered to be a surrogate marker for multidrug-resistant Mycobacterium tuberculosis (MDRTB).

Objective: The present study was undertaken to evaluate the drug resistance profile and the resistance-conferring mutations of MDR-TB species in a tertiary care hospital, Central Kerala.

Methods: Clinical specimens $(n=404)$ were subjected to Ziehl-Neelsen $(Z-N)$ staining and culture and drug susceptibility testing (DST) using liquid (BD BACTEC ${ }^{\text {TM }}$ Micro MGIT ${ }^{\text {TM }}$ \{Mycobacteria Growth Indicator tube). RIF resistant isolates ( $\mathrm{n}=5$ ) detected phenotypically along with randomly selected RIF sensitive isolates $(n=5)$ were randomly selected and $r p o B$ gene of all the amplified strains was sequenced and mutations analysed.

Results: Out of 404 samples from clinically suspected cases of $M$. tuberculosis, Mycobacteria was grown in 48 (11.9\%) samples. Amongst the forty-eight $M$. tuberculosis isolates, $41.6 \%$ were sensitive to all five drugs and $6.2 \%$ were resistant to all five drugs. Of the total of 48 culture-positive TB cases, one (2.08\%) was found to be MDR-TB and additional resistance was observed in one $(2.08 \%$ ) isolate. Of the ten isolates (five RIF resistant isolates detected phenotypically along with five RIF sensitive isolates) which was amplified by PCR, M. tuberculosis DNA was not detected by PCR in one of the isolates. Mutations detected occurred in codon 531 with Ser $\rightarrow$ Leu substitution.

Conclusion: Molecular methods along with conventional detection methods help in understanding the transmission dynamics of tuberculosis and could be used as a tool in controlling the transmission of tuberculosis and thereby achieving the elimination of tuberculosis by 2030.

Key Words: Rifampicin, M. tuberculosis, Mutation

\section{INTRODUCTION}

Tuberculosis (TB) is one of the major infectious diseases and is the leading cause of death from a single infectious agent. ${ }^{1}$ According to the world TB report (2019) by World Health Organization (WHO), an estimated 10 million new active TB diseases cases and 1.2 million deaths occurred in $2018 .{ }^{2} \mathrm{In}-$ dia ranks first among the eight high burden countries which contribute to the $2 / 3^{\text {rd }}$ of the global TB burden. ${ }^{2}$ In 2018 , there were about half a million new cases of rifampicin-resistant TB out of which $78 \%$ had multidrug-resistant TB. ${ }^{2}$ Even though there is an increase in the notification rates, there is still a gap between the number of new cases and the estimated cases reported. This gap is mainly due to the under-reporting of detected cases or under-diagnosis. Also in resource-poor settings like India, the detection and treatment of multidrug-resistant tuberculosis (MDR-TB) is all the more difficult especially due to the high expenses and more toxic treatment regimens which lead to higher rates of clinical failure and disease relapse. ${ }^{3}$

The evolution of drug resistance in clinical $M$. tuberculosis strains is mainly due to chromosomal mutations in target genes. Other mechanisms include disruption of prodrug activation and the activation of efflux pump. World health organisation (WHO) has issued a recommendation that drug susceptibility testing (DST) of M. tuberculosis isolates should be carried out for all patients with TB to aid treatment

\section{Corresponding Author:}

Dr. Seema Oommen, Specialist Microbiologist, Lifecare Hospital, and Molecular Biologist, Burjeel Medical City, Abu Dhabi, United Arab Emirates; Email: seema.oommen@gmail.com

ISSN: 2231-2196 (Print)

Received: 11.09 .2020
ISSN: 0975-5241 (Online)

Revised: 23.10 .2020
Accepted: 02.12 .2020
Published: 03.03 .2021 
plans and to increase the outcome. ${ }^{3,4}$ The main mechanisms of resistance leading to drug resistance include drug target alteration, overexpression of drug target, disruption of prodrug activation and the activation of efflux pump. ${ }^{4}$ Gene mutations are usually associated with resistance to specific drugs used in treating M. tuberculosis. ${ }^{5}$ Rifampicin is one of the most potent first-line drugs available for TB treatment. Early detection of rifampicin resistance is of particular importance since $>90 \%$ of rifampicin-resistant isolates are also resistant to isoniazid. Therefore, the detection of rifampicin resistance represents a valuable surrogate marker for MDRTB. ${ }^{6}$ Resistance to rifampicin has been demonstrated as a result of single or multiple mutations in the rpoB gene, the RNA polymerase $\beta$-subunit-encoding gene. ${ }^{7}$ The $\beta$ subunit of RNA polymerase is coded by the $r p o B$ gene. Sequencing studies have shown that $90-95 \%$ of rifampicin-resistant $M$. tuberculosis isolates have a mutation within an 81-bp hot-spot region (codons 507-533; Escherichia coli numbering) of the rpoB gene which is the core region (Rifampicin Resistance Determining Region, RRDR). ${ }^{8}$ Molecular techniques are used to differentiate $M$. tuberculosis strains and are important tools in public health issues related to TB outbreaks and to unravel the transmission patterns. ${ }^{5}$ M.tuberculosis complex (MTBC) lineages have differences in virulence, transmissibility, and capacity of acquiring drug-resistance conferring mutations. ${ }^{9}$ One of the most used genotyping methods is spacer oligonucleotide typing (spoligotyping), which is based on the polymorphism of direct repeat (DR) locus present within $M$. tuberculosis. The DR contains 36-bp well-conserved repeat sequences interspersed with 34-41-bp nonrepetitive spacers and therefore used to differentiate strains. ${ }^{10}$ The spoligotyping method was also the basis for making the largest genotype database for $M$. tuberculosis, containing a global distribution and phylogenetic analysis for worldwide spoligotype. ${ }^{11}$

The importance of molecular typing of M. tuberculosis has been useful in the outbreak investigations in health care settings, as it helps to understand the transmission dynamics of M. tuberculosis strains and their distribution at a particular region/area. ${ }^{12}$ This study was carried out to investigate the predominant $M$. tuberculosis strains responsible for causing tuberculosis in a tertiary care hospital in Kerala using spoligotyping and identify drug resistance gene and its mutations associated, prevalent in M. tuberculosis strains. With the increased number of cases and many of them becoming multidrug-resistant (MDR) or extensively drug-resistant TB (XDR-TB), a better knowledge of the molecular mechanisms of antitubercular drug-resistance will have a significant impact on the improvement of rapid detection of drug resistance, especially when using molecular formats for detection of drug resistance. As such, little information is available on the aetiology of MDR-TB in the area where the present study is conducted. Therefore, the present study was undertaken to determine the characteristics, and evaluate the drug resistance profile of MDR-TB species and to investigate the drug resistant conferring mutations and to ascertain their epidemiological linkages using molecular genotyping.

\section{MATERIALS AND METHODS}

A total of 404 specimens were obtained from suspected cases of tuberculosis (TB) and from patients who were previously treated for TB from different wards and outpatient departments of a 1200 bedded tertiary care centre located in Central Kerala for a period of two years from November 2015 to October 2017. The institutional ethical committee clearance was obtained for the study (PIMSRC/E1/388A/44/2015 dated 29-10-2015). Consecutive pulmonary and extrapulmonary specimens (except blood) were collected from patients, suspected of TB, irrespective of their age and gender from various clinical departments like pulmonary medicine, general medicine, surgery, and paediatrics in sterile; leak-proof containers. The samples were processed in the mycobacteriology laboratory within $48 \mathrm{~h}$ of receipt of specimen. All specimens were subjected to direct smear microscopy using Ziehl-Neelsen (ZN) staining method followed by N-acetylL-cysteine and sodium hydroxide (NALC-NaOH) digestion, decontamination technique. Decontamination is not required for sterile body fluid. After decontamination, one drop of the specimen was taken and smear is prepared. The liquid culture medium used was the BBL Mycobacteria Growth Indicator Tube (MGIT BD BACTEC ${ }^{\mathrm{TM}}$ ) containing modified Middle Brook $7 \mathrm{H} 9$ broth $(7 \mathrm{~mL})$, to which an enrichment supplement, as well as a mixture of antimicrobials consisting of polymyxin $\mathrm{B}$, amphotericin $\mathrm{B}$, nalidixic acid, trimethoprim, and azlocillin, was added. The MGIT tubes along with the solid media were incubated at $37^{\circ} \mathrm{C}$. The tubes were examined daily up to 8 weeks of incubation in a $365 \mathrm{~nm}$ wavelength UV light source fluorescence detector (BACTEC ${ }^{\mathrm{TM}}$ Micro MGIT device). To validate the test a positive and a negative control tube were included with every batch of test tubes examined. The tubes were checked for the presence of granular appearance and fluorescence which indicates growth. On solid media, growth was observed as rough, cream coloured colonies. All positive tubes were confirmed for the presence of AFB by Z-N staining and were subcultured on a blood agar plate to rule out contamination. The smears were observed for the presence of AFB, as well as for the presence of cording a characteristic feature of $M$. tuberculosis complex (MTBC). MTBC isolates were further confirmed using BD MGIT MTBC identification test (TBC ID).

The MTBC isolates were further subjected to drug susceptibility testing using the drugs streptomycin $(1 \mu \mathrm{g} / \mathrm{mL})$ (STR), isoniazid $(0.1 \mu \mathrm{g} / \mathrm{mL})(\mathrm{INH})$, rifampicin $(1 \mu \mathrm{g} / \mathrm{mL})$ (RIF), ethambutol $(5 \mu \mathrm{g} / \mathrm{mL})(\mathrm{ETH})$ and also pyrazinamide (100 $\mu \mathrm{g} / \mathrm{mL}$ ) (PZA). BD BACTEC MGIT 960 SIRE kits were used for drug susceptibility testing. Five RIF resistant iso- 
lates detected phenotypically along with five RIF sensitive isolates were randomly selected and were analysed. The point mutations within the $81 \mathrm{bp}$ RRDR were detected using PCR and DNA sequencing methods and the sequences were compared with wild type $\mathrm{M}$. tuberculosis $(\mathrm{H} 37 \mathrm{Rv})$ strain to check for any mutations within the hotspot region. Finally, the amplicons were subjected to spoligotyping.

\section{DNA EXTRACTION, PCR AND SEQUENCING}

DNA extraction was performed as per the manufacturer's instructions using the QIAamp DNA Mini Kit (Qiagen). Samples from the broth culture were subjected to centrifugation and the pellet dissolved in minimum volume in buffer/MilliQ for further Qiagen DNA Extraction method. The isolated mycobacterial DNA was subjected to PCR amplification using species-specific primers. The DNA was measured using the Nanodrop ND-1000 Spectrophotometer (Thermofisher) and visualized on $0.8 \%$ agarose gel stained with ethidium bromide. The $81 \mathrm{bp}$ hot spot, flanking regions of $r p o B$ gene of $M$. tuberculosis was amplified using polymerase chain PCR primers rpoB F forward - 5'- GGATCAGCTCGCCGACCGTA 3' and PCR primers rpoB R Reverse - 5'TACGGCGTTTCGATGAACC-3' were used.

Applied Biosystem thermal cycle was used for the PCR reaction. The conditions set for PCR reaction were, initial denaturation at $95^{\circ} \mathrm{C}$ for $2 \mathrm{~min} ; 34$ cycles of $95^{\circ} \mathrm{C}$ for $40 \mathrm{sec}$, $55^{\circ} \mathrm{C}$ for $50 \mathrm{sec}$ and $72^{\circ} \mathrm{C}$ for $30 \mathrm{sec}$; and final elongation at $72^{\circ} \mathrm{C}$ for $7 \mathrm{~min}$, followed by holding at $4^{\circ} \mathrm{C}$. The positive control used was M. tuberculosis $\mathrm{H} 37 \mathrm{Rv}$ and negative control used was nuclease-free water. Post amplification, the samples were run on a $1.5 \%$ agarose gel and visualization was done under a UV Transilluminator. After obtaining the desired amplification, the PCR products are subjected to Exo SAP purification. Post ExoSAP purification of the PCR products, cycle sequencing was carried out using the ABI Big Dye Terminator v.3.0 (Applied biosystems) kit. Products were resolved by electrophoresis on an ABI 3730xl capillary sequencer. The $411 \mathrm{bp}$ amplicons were purified and sequenced using the primer $r p o B \mathrm{~F}$ or rроB $\mathrm{R}$ and the sequences were aligned with gi|448814763:759807-763325 M. tuberculosis $\mathrm{H} 37 \mathrm{Rv}$, reference sequence for the screening of rроB gene mutations.

\section{RESULTS}

A total of 404 specimens were obtained from suspected cases of tuberculosis (TB) and from patients who were previously treated for TB from different wards and outpatient departments during the study period.

Of the 404 samples collected, 188 (46.5\%) samples were from pulmonary TB suspected patients and $216(53.5 \%)$ from extrapulmonary TB (EPTB) suspects. These 404 specimens were processed by smear and further by culture on to Micro MGIT and Lowenstein- Jensens (L-J) medium. Among the 188 pulmonary samples, $27(14.4 \%)$ specimens were found to be positive for M. tuberculosis complex (MTBC), and out of the 216 extrapulmonary samples processed, $21(9.7 \%)$ were found to be positive for MTBC by the Micro MGIT culture. Thus, of the 404 samples processed, a total of $11.9 \%$ (48/404) were found to be positive for TB.

Amongst the forty-eight $M$. tuberculosis isolates, $41.6 \%$ $(\mathrm{n}=20)$ were sensitive to all five drugs and $6.2 \%(\mathrm{n}=3)$ were resistant to all five drugs. The highest rate of mono resistance was towards streptomycin (STR) $(n=7,14.9 \%)$, followed by isoniazid $(\mathrm{INH})(\mathrm{n}=6,12.5 \%)$ and pyrazinamide $(\mathrm{PZA})(\mathrm{n}=4$, $8.3 \%$ ). Among the total of 48 culture-positive TB cases, one (2.1\%) was found to be MDR-TB and additional resistance (resistance to STR, INH, RIF and PZA) was observed in one (2.1\%) isolate. The MDR isolates obtained was from a pulmonary TB patient. Around $12.5 \%(\mathrm{n}=6)$ of the total culturepositive TB cases were poly-resistant.

Five RIF resistant isolates detected phenotypically along with five randomly selected RIF sensitive isolates was subjected to M. tuberculosis DNA isolation. The isolated mycobacterial DNA was subjected to PCR amplification using species-specific primers. A clear band in the agarose gel at $411 \mathrm{bp}$ confirmed the presence of $M$. tuberculosis. Of the ten isolates subjected to PCR amplification, M. tuberculosis DNA was not detected by PCR in one of the resistant isolates (Figure 1). The reason may be or that the isolate could be non-tuberculous mycobacteria which cannot be detected by $M$. tuberculosis species-specific primers. This isolate was considered as M. tuberculosis complex phenotypically.

Five phenotypically RIF-resistant isolates and four RIF sensitive isolates were tested for mutations in the $r p o B$ gene. DNA sequence analysis revealed that $60 \%(3 / 5)$ strains of RIF-resistant showed rpoB gene mutation versus $40 \%(2 / 5)$ strains to have no mutation. The only rрoB gene mutation described by sanger sequencing occurred in codon 531 in three isolates with Ser $\rightarrow$ Leu substitution (TCG $\rightarrow$ TTG) (Figure 2). Two isolates, although resistant to RIF, did not show any mutation.

\section{DISCUSSION}

Worldwide, resistance to standard anti-tuberculosis drugs is a major constrain in the control and treatment of tuberculosis. ${ }^{13,14}$ Accurate determination of the species and strains is critical in inpatient management. A total of 404 specimens were obtained for culture from suspected pulmonary and extrapulmonary TB patients of which $188(46.5 \%)$ was from pulmonary TB suspects and 216 (53.5\%) from extrapulmonary TB suspects. In this study samples $(\mathrm{n}=404)$ were received 
in the laboratory and were processed for Zeihl-Neelsen staining and liquid and solid culture simultaneously. The positivity rates using smear microscopy was $5.0 \%$ (pulmonary and EPTB specimens) when compared to liquid culture method which was $11.9 \%$ and solid culture method which detected $8.7 \%$ of TB cases.

Drug-resistant TB is still a major public health problem. In the present study, DST was carried out for all first-line anti-tubercular drugs - streptomycin (STR) $(1.0 \mu \mathrm{g} / \mathrm{mL})$, isoniazid (INH) $(0.1 \mu \mathrm{g} / \mathrm{mL})$, rifampicin (RIF) $(1 \mu \mathrm{g} / \mathrm{mL})$, ethambutol (ETB) $(5 \mu \mathrm{g} / \mathrm{mL})$ and pyrazinamide (PZA) $(100$ $\mu \mathrm{g} / \mathrm{mL})$. It was found that $41.7 \%(\mathrm{n}=20 / 48)$ isolates were sensitive to all five drugs. Similar observations from North India by Sethi et al., (2012), who reported $47.5 \%$ pan-sensitive isolates. ${ }^{15}$ Resistance to one drug was observed in 17/48 (35.4\%) of the isolates in the present study. This is similar to a study conducted in China by Ning-ning Tao et al., (2017), where resistance to a single drug was found to be $24.1 \% .^{16}$ In the present study, only one isolate $(2.8 \%)$ was identified as MDR phenotypically as per the definition. Globally, 3.5\% of new cases and $18 \%$ of previously treated cases had MDR/ RR-TB (1). In 2017, there were an estimated 558,000 (range: 483,000-639,000) incident cases of MDR/RR-TB. The countries with the largest numbers of MDR/RR-TB cases ( $47 \%$ of the global total) were China, India and the Russian Federation. ${ }^{1}$

The use of molecular methods to identify mutations associated with drug resistance has drastically decreased the turnaround time and is highly specific to phenotypic DST. High burden of drug-resistant TB makes a serious problem the TB control in India. Treatment of TB infection relies primarily on the use of first-line drugs including Isoniazid (INH) and rifampicin (RIF) with ethambutol (ETB), streptomycin (STR) and pyrazinamide (PZA). ${ }^{17}$ RIF due to its excellent bactericidal activity is considered to be the efficient drug in the treatment regimen for tuberculosis ${ }^{18}$. Whereas mono resistance to INH is quite common, mono resistance to RIF is rare. Instead, RIF resistance occurs most often in strains that are also resistant to INH; thus, RIF resistance can be used as a surrogate marker for MDR..$^{19,20}$

DNA sequencing of $r p o B$ gene in MDR TB has an added advantage of increased knowledge of mutation profile of $r p o B$ gene, which may help in the development of a screening protocol, relevant to the geographical area, for early detection of MDR TB. ${ }^{21}$

The mechanism of action of RIF is to inhibit mycobacterial transcription by targeting DNA-dependent RNA polymerase. RIF resistance in M. tuberculosis complex strains emerge as a result of point mutations, or small deletions, or insertions, in a limited region of the gene encoding for the $r p o B$ gene (codons 507-533) encoding 27 amino acids. ${ }^{22}$ Mutations of the RIF-resistant $M$. tuberculosis isolates are located in an 81-bp core region, the RIF resistance determining region [RRDR]), of the rpoB gene in about $95-98 \%$ of RIF resistant strains. ${ }^{22-24}$ Two of the rifampicin-resistant isolates obtained phenotypically did not show any mutation in the RRDR region. This may be due to the presence of other rare rpoB mutations or another mechanism of resistance to rifampicin ${ }^{25}$.

Five phenotypically RIF resistant isolates detected along with the five RIF sensitive isolates were randomly selected and subjected to M.tuberculosis deoxyribonucleic acid (DNA) isolation and amplification using species-specific primers. Of the ten isolates subjected to PCR, amplification of M. tuberculosis was not observed in one isolate, which was also incidentally resistant to all five $1^{\text {st }}$ line drugs including RIF. The isolate was from a diabetic patient who had chronic pain in the left leg and eventually led to the loss of sensation. The left leg had a wound and it was infected and the pus was sent for a culture which grew Mycobacterium spp. by about 40 days of incubation in liquid culture. This isolate was identified as M. tuberculosis complex (MTBC) by phenotypic species identification method, the MGIT TBC Identification Test (Becton Dickinson Diagnostic Instrument Systems, Sparks, MD. The reason for the absence of amplification may be because, though the assay demonstrates $100 \%$ sensitivity as reported, the specificity is between $95.2-100 \%{ }^{26}$. The isolate was a non-tuberculous mycobacterium and was falsely reported positive for MTBC by this test. Fortunately, we did not report this isolate as M.tuberuclosis complex due to the suspicion that it could be non-tuberculous mycobacteria, especially after the drug resistance pattern. Thus, it is important not to solely rely on the rapid tuberculosis identification methods for identification of M. tuberculosis complex especially if the isolate appears to be resistant to all five drugs. Further speciation of the isolate would then become necessary.

In the present study, the only mutation detected was observed at codon 531 (3/5) (TCG-TTG) and was observed in three out of four RIF resistant isolates. This is similar to the reports from various parts of India by Makadia et al., (2012), where the most common sites of mutation were at codon 531 (TCG-TTG), 526 (CAC- TAC) and 516 (GACGTC, AAC), with the frequency of mutation at 66.7, 20 and $13.3 \%$, respectively ${ }^{21}$. Codons 531, 526, 516 and 511 are reported as the most frequent mutations in the rрoB fragment worldwide. ${ }^{27-29}$ While TTG at codon 531 and CCG at codon 511 are the dominant mutated alleles, codon 526 and codon 516 show large numbers of allelic variations ${ }^{[29]}$. Various studies show that mutations at different codons of rpo $B$ could be associated with different levels of RIF resistance. The mutation at codon 531 or 526 was associated with highlevel resistance to RIF (minimum inhibitory concentration [MIC] $>64 \mathrm{mg} / \mathrm{mL}$ ) and high-level cross-resistance to all rifamycins, whereas mutation at codon 516 was associated with medium-level resistance to RIF (MIC $=32 \mathrm{mg} / \mathrm{mL}$ ), but 
susceptible to rifabutin ${ }^{30}$. Thus, our three isolates showed high-level resistance to rifampicin.

A study conducted in Tamil Nadu, India, by Deepa et al., (2005), reported that the most commonly reported missense mutation was at codon 531 (Ser $\rightarrow$ Leu) in two isolates and one at 526. Rest two samples did not show any mutation in RRDR region. ${ }^{31}$ Similar observations were made in the present study, where one of the phenotypically detected RIF resistant isolate did not show any mutations on sequencing. A study in Brazil by Andreia et al., (2000), reported that the codons most frequently affected were 531 (TCG-TTG), 526 (CAC-TAC) and 516 (GAC-GTC) with frequencies of 54,21 and $7 \%$, respectively. About $18 \%$ of samples did not show any mutation in RRDR region. ${ }^{32}$ Finding of no mutations in the RRDR of rpoB gene of the single isolate which was phenotypically resistant indicates mutations outside the 81-bp segment (RRDR) of rpoB or additional molecular mechanisms that may be involved in RIF resistance of $M$. tuberculosis like permeability barrier or membrane proteins acting as drug efflux pumps..$^{33}$ This may be the reason for no mutations in one out of the four isolates which were showing RIF resistance using phenotypic methods.

Studies show that multiple mutations can occur at different nucleotide in the same codons. ${ }^{21}$ It is also observed that some samples had multiple mutations in RRDR region of $r p o B$ gene. ${ }^{21}$ No such findings were seen in the present study.

\section{CONCLUSIONS}

Molecular methods help in understanding the transmission dynamics of tuberculosis and could be used as a tool in the current control programme locally as well as internationally. It also helps in designing the primers and probes for diagnostic detection of resistance. Genotyping of $M$. tuberculosis strains together with epidemiological studies may throw a light regarding the transmission of the strain

\section{ACKNOWLEDGEMENTS}

Authors acknowledge the immense help received from the scholars whose articles are cited and included in references to this manuscript. The authors are also grateful to authors/ editors/publishers of all those articles, journals and books from where the literature for this article has been reviewed and discussed. The authors are thankful to Mapmygenome, Hyderbad for helping with carrying out the molecular studies.

\section{Financial Support: None}

\section{Conflict of Interest: Nil}

\section{REFERENCES}

1. World Health Organization. Global TB report [Internet]. 2018. Available from: WHO/CDS/TB/2018.20

2. Tb D. Global Tuberculosis Tuberculosis. WHO/CDS/ TB/2019. 2019.

3. Ahmad S, Mokaddas E. Current status and future trends in the diagnosis and treatment of drug-susceptible and multidrug-resistant tuberculosis. J Infect Public Health 2014;7(2):75-91.

4. Miotto P, Zhang Y, Cirillo DM, Yam WC. Drug resistance mechanisms and drug susceptibility testing for tuberculosis. Respirology 2018;23(12):1098-1113.

5. De Freitas FAD, Bernardo V, Gomgnimbou MK, Sola C, Siqueira HR, Pereira MAS, et al. Multidrug-resistant Mycobacterium tuberculosis: A retrospective $\mathrm{katG}$ and $\mathrm{rpoB}$ mutation profile analysis in isolates from a reference centre in Brazil. PLoS One. 2014;9(8):1-11.

6. Prammananan T, Cheunoy W, Taechamahapun D, Yorsangsukkamol J, Phunpruch S, Phdarat P, et al. Distribution of rpoB mutations among multidrug-resistant Mycobacterium tuberculosis (MDRTB) strains from Thailand and development of a rapid method for mutation detection. Clin Microbiol Infect 2008;14(5):446-453.

7. Telenti A, Imboden P, Marchesi F, Lowrie D, Cole S, Colston $\mathrm{MJ}$, et al. Detection of rifampicin-resistance mutations in Mycobacterium tuberculosis. Lancet 1993;341:647-650.

8. Kapur V, Li LL, Iordanescu S et al. Characterization by automated DNA sequencing of mutations in the gene (rpoB) encoding the RNA polymerase beta subunit in rifampin-resistant Mycobacterium tuberculosis strains from New York City and Texas. J Clin Microbiol 1994;32(4):1095-1098.

9. Molina-Moya B, Abdurrahman ST, Madukaji LI, Gomgnimbou MK, Spinasse L, Gomes-Fernandes M, et al. Genetic characterization of Mycobacterium tuberculosis complex isolates circulating in Abuja, Nigeria. Infect Drug Resist 2018;11:1617-1625.

10. Prim RI, Schörner MA, Senna SG, Nogueira CL, Figueiredo ACC, de Oliveira JG, et al. Molecular profiling of drug resistant isolates of Mycobacterium tuberculosis in the state of Santa Catarina, southern Brazil. Mem Inst Oswaldo Cruz 2015;110(5):618-623.

11. Brudey K, Driscoll JR, Rigouts L, Prodinger WM, Gori A, Alhajoj SA, et al. Mycobacterium tuberculosis complex genetic diversity : mining the fourth international spoligotyping database ( SpolDB4 ) for classification, population genetics and epidemiology. BMC Microbiol 2006;17:1-17.

12. Barnes PF. Molecular epidemiology of tuberculosis. N Eng J Med 2003;349:1149-1156.

13. Romero BA, Aranaz L, Juan JA, lvarez J, Bezos A, Mateos EG. Molecular epidemiology of multidrugresistant Mycobacterium Bovis isolates with the same spoligotyping profile as isolates from animals. J Clin Microbiol 2006;44(9):3405-3408.

14. Zignol M, Hosseini MS, Wright A, van Weezenbeek CL, Nunn $\mathrm{P}$, Watt CJ, et al. Global incidence of multidrugresistant tuberculosis. J Infect Dis 2006;194:479-485.

15. Sunil S, Manisha B, Chatterjee SS, Abhishek M, Singh SKK, Meera S. Susceptibility pattern among pulmonary and extrapulmonary isolates of Mycobacterium tuberculosis in north India. African J Microbiol Res 2012;6(15):3696-3699.

16. Rahman M, Kamal SM, Mohammed FR, Alam MB. Anti-tuberculosis drug resistance pattern among the different category of tuberculosis patients. J Med 2009;10:45-47.

17. World Health Organization. Treatment of tuberculosis: Guidelines for national programmes. 3rd Edition, WHO, Geneva. 2003. 
18. Burman WJ, Gallicano K, Peloquin C. Comparative pharmacokinetics and pharmacodynamics of the rifamycin antibacterials. Clin Pharmacokinet 2001;40, 327-341.

19. Alcaide F, Benítez MA, Escribà JM, Martín R. Evaluation of the BACTEC MGIT 960 and the MB/BacT systems for recovery of mycobacteria from clinical specimens and for species identification by DNA AccuProbe. J Clin Microbiol 2000;38(1):398-401.

20. Yon J, Ryu M. Diagnosis of Pulmonary Tuberculosis: Recent Advances and Diagnostic Algorithms. Tuberc Respir Dis 2015;78(2):64-71.

21. Makadia JS, Jain A, Kumar S, Sherwal PBL, Khanna A. Emerging Trend of Mutation Profile of rpoB Gene in MDR Tuberculosis, North India 2012;27(4):370-374.

22. Somoskovi A, Parsons LM, Salfinger M. The molecular basis of resistance to isoniazid, rifampin, and pyrazinamide in Mycobacterium tuberculosis. Respir Res 2001;2:164-168.

23. Simon S. Drug resistance in Mycobacterium tuberculosis: a molecular perspective. Madjalah Kedokt Indones 2003;4:26-35.

24. Hillemann D, Weizenegger M. Use of the genotype MTBDR assay for rapid detection of rifampin and isoniazid resistance in Mycobacterium tuberculosis complex isolates. J Clin Microbiol 2005;43:3699-703.

25. Qazi O, Rahman H, Tahir Z, Qasim M, Khan S, Ahmad AA, et al. Mutation pattern in rifampicin resistance determining region of rpoB gene in multidrug-resistant Mycobacterium tuberculosis isolates from Pakistan. Int J Mycobacteriol 2014;3(3):173-177.

26. Gaillard T, Fabre M, Martinaud C, Vong R, Brisou P. Assessment of the SD Bioline Ag MPT64 Rapid and the MGIT TBc

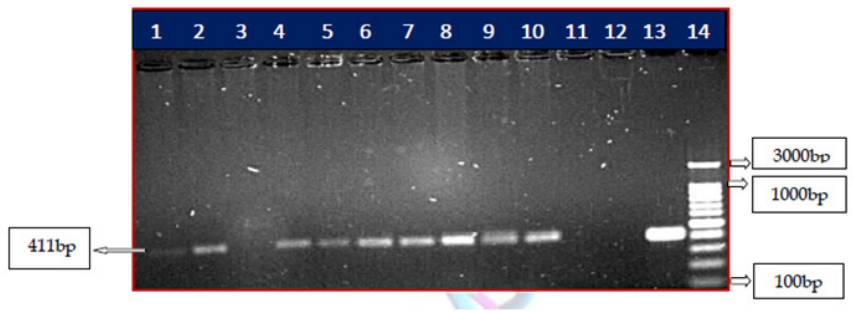

Figure 1: PCR amplification of $r p o B$ gene of phenotypic MDRTB isolates. PCR product of $r p o B$ was resolved on $1.5 \%$ agarose gel. Lane 1-10-shows the amplification of 10 phenotypic sensitive and resistant isolates. Lane 11-Blank, Lane 12-negative control, lane 13- positive control M. tuberculosis H37RV strain ,Lane 14- 100 bp ladder, product size of 411 bp. identification tests for the diagnosis of tuberculosis. Diagn Microbiol Infect Dis 2011;70(1):154-156.

27. Ahmad S, Mokaddas E, Fares E. Characterization of rpoB mutations in rifampin-resistant clinical Mycobacterium tuberculosis isolates from Kuwait and Dubai. Diagn Microbiol Infect Dis 2002;44(3):245-252.

28. Bakonyte D, Baranauskaite A, Cicenaite JS, Stakenas P. Mutations in the rpoB gene of rifampin-resistant Mycobacterium tuberculosis clinical isolates from Lithuania. Int J Tuberc Lung Dis 2005;9:936-938.

29. Cavusoglu C, Hilmioglu S, Guneri S, Bilgic A. Characterization of rpoB Mutations in Rifampin-Resistant Clinical Isolates of Mycobacterium tuberculosis from Turkey by DNA Sequencing and Line Probe Assay. J Clin Microbiol 2002;40(12):4435-8.

30. Dalal A, Pawaskar A, Das M, Desai R, Prabhudesai P, Chhajed P et al. Resistance patterns among multidrug-resistant tuberculosis patients in greater metropolitan Mumbai: trends over time. PLoS One 2015;10(1):e0116798.

31. Deepa P, Therese KL, Madhavan HN. Detection and characterization of mutations in rifampicin resistant mycobacterium tuberculosis clinical isolates. Indian J Tuberc 2005;6:132-136.

32. Andreia RM, Lucia M, Rossetti R, Ribeiro MO ZA. Mutations in rpoB gene of multidrug resistant Mycobacterium tuberculosis isolates from Brazil. J Clin Microbiol 2000;38:3119-3122.

33. Banik A, Das N, Wihiwot V, Lyngdoh ACP, Dut V. Prevalence and first-line drug sensitivity trends of Mycobacterium tuberculosis at a tertiary center in North-East India. Egypt J Chest Dis Tuberc 2018;67:32-37.

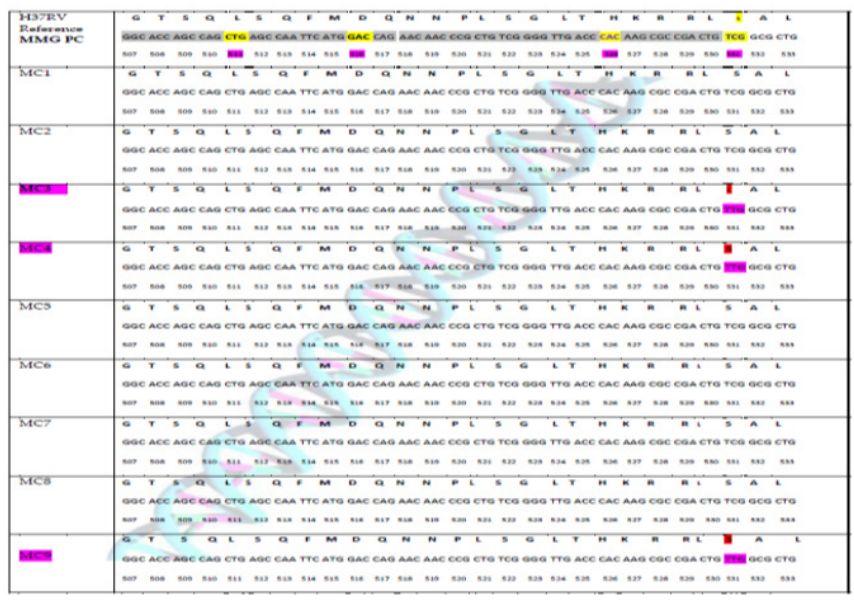

Figure 2: Distribution of mutations in the rpoB gene among MDR isolates. The figure shows the results of DNA sequencing compared with reference DNA sequence of RRDR of rpoB gene of $\mathrm{M}$. tuberculosis. The observed mutation is in codon 531 and is shown in three isolates which is rifampicin resistant. No mutation in RRDR region of rpoB gene was found in 2 isolates though it was rifampicin resistant. 